\title{
Porosity detection in electron beam-melted Ti-6Al-4V using high- resolution neutron imaging and grating-based interferometry
}

\author{
Adam J. Brooks ${ }^{1} \cdot$ Jinghua Ge $^{2} \cdot$ Michael M. Kirka ${ }^{3} \cdot$ Ryan R. Dehoff $^{3} \cdot$ \\ Hassina Z. Bilheux ${ }^{4} \cdot$ Nikolay Kardjilov $^{5} \cdot$ Ingo Manke $^{5} \cdot$ Leslie G. Butler $^{1}$
}

Received: 21 December 2016/ Accepted: 28 April 2017/Published online: 10 May 2017

(C) Springer International Publishing Switzerland 2017

\begin{abstract}
A high-resolution neutron tomography system and a grating-based interferometer are used to explore electron beam-melted titanium test objects. The high-resolution neutron tomography system (attenuation-based imaging) has a pixel size of $6.4 \mu \mathrm{m}$, appropriate for detecting voids near $25 \mu \mathrm{m}$ over a $(1.5 \mathrm{~cm})^{3}$ volume. The neutron interferometer provides dark-field (small-angle scattering) images with a pixel size of $30 \mu \mathrm{m}$. Moreover, the interferometer can be tuned to a scattering length, in this case, $1.97 \mu \mathrm{m}$, with a field-of-view of $(6 \mathrm{~cm})^{3}$. The combination of high-resolution imaging with grating-based interferometry provides a way for nondestructive testing of defective titanium samples. A chimney-like pore structure was discovered in the attenuation and dark-field images along one face of an electron beam-melted (EBM) Ti-6Al$4 \mathrm{~V}$ cube. Tomographic reconstructions of the titanium samples are utilized as a source for a binary volume and for skeletonization of the pores. The dark-field volume shows
\end{abstract}

Adam J. Brooks

abroo38@1su.edu

Jinghua Ge

jinghuage@cct.lsu.edu

Michael M. Kirka

kirkamm@ornl.gov

Ryan R. Dehoff

dehoffrr@ornl.gov

Hassina Z. Bilheux

bilheuxhn@ornl.gov

Nikolay Kardjilov

kardjilov@helmholtz-berlin.de

Ingo Manke

manke@helmholtz-berlin.de

Leslie G. Butler

lbutler@1su.edu features with dimensions near and smaller than the interferometer auto-correlation scattering length.

Keywords Neutron interferometry · Electron beam melting · Additive manufacturing · Porosity · Tomography

\section{Introduction}

Ti-6Al-4V additive manufacturing (AM) has played a key role in modern scientific exploration, from biocompatibility studies with mouse fibroblast cells [1] to dental implants [2]. As the role of AM grows larger, so does the need for understanding the effects of printing methods on materials. A recent model comparing the differences between electron beam melting and selective laser melting of Ti-6Al-4V serves as a guide as to how the microstructure can be controlled through AM [3]. While modeling may be

1 Department of Chemistry, Louisiana State University, Baton Rouge, LA 70803, USA

2 Center for Computation and Technology, Louisiana State University, Baton Rouge, LA 70803, USA

3 Manufacturing Demonstration Facility, Oak Ridge National Laboratory, Oak Ridge, TN 37831, USA

4 Chemical and Engineering Materials Division, Oak Ridge National Laboratory, Oak Ridge, TN 37831, USA

5 Helmholtz Center Berlin for Materials and Energy, 14109 Berlin, Germany 
valuable, it does not reveal any information as to whether a desired manufactured object was printed correctly.

One simple way to look at this problem is through imaging. X-ray and neutron imaging provide a way to visualize $3 \mathrm{D}$ printed objects without the need for destruction of samples. Traditional imaging involves 2D radiography, where a wavefront from a source (X-ray or neutron) travels in a designated path and interacts with a sample placed in the beam path at a certain distance. Alterations to the wavefront are detected through attenuation/absorption images and the object can then be reconstructed.

In comparison to the one dataset provided from attenuation-based radiography, grating-based interferometry generates three datasets: traditional attenuation/absorption, differential phase contrast, and dark-field (synonymous with small-angle scattering). With many different methods of X-ray and neutron interferometry developed over the years (Michelson, Talbot-Lau [4], and Far-Field [5]), of interest here is the Talbot-Lau interferometer. In a TalbotLau interferometer, a source grating, G0, adds coherence to an incoming wavefront (either X-rays or neutrons). The wave proceeds along the beam path and interacts with a phase grating, G1, which alters the wave by a certain period that is then propagated further downstream. Intensity changes of the beam can be detected using an analyzer grating, G2, after an object is placed in the beam path.

While interferometry is a well-known technique, one recent hindrance was grating fabrication. Until 2006, grating fabrication was only well established for X-ray interferometry (X-ray lithography) [6]. Challenges with gadolinium sputtering for source and analyzer gratings limited the production of neutron gratings [7] until Kim/ Lee began filling silicon gratings with Gadox powder to produce the same optical properties [8]. Interferometry has now become a valuable tool for neutron imaging of materials.

With two main types of imaging (X-rays and neutrons), questions arise as to which is the more viable imaging option for AM materials. X-ray computed tomography (XCT) has proven valuable for observing polymer AM pores [9]. Micro X-ray CT ( $\mu$ XCT) offers very high spatial resolution (as low as $5 \mu \mathrm{m}$ ) for observing Ti-6Al-4V lack of fusion, keyhole, and gas porosity [10, 11]. While higher spatial resolution is preferred, the high absorbance of $\mathrm{X}$-rays by metals and a small sample size requirement for $\mu \mathrm{XCT}$ limits the reproducibility of imaging large-scale samples. In comparison, neutron imaging provides bulk sensitivity and can show strain distribution in Inconel 625 [12]. In the case of Ti-6Al-4V samples with micron-sized spacing, the interaction of neutrons with the metal results in an expected phase shift of the beam and an altered signal in the dark-field. In comparison to attenuation imaging, where resolution is limited down to 15 microns, the darkfield signal can reach as low as 1-micron resolution [13].

The possibility exists for one imaging method, such as attenuation, to measure defects in AM samples undetected with another imaging modality. Due to the chance of porosity defects occurring at multiple length scales (nanometer and micrometer), the need exists for performing high-resolution attenuation imaging and grating-based interferometry. The interferometry can detect changes in the small-angle scattering (dark field) while high-resolution imaging can magnify noticeable features through attenuation. For a several cm thick AM Ti-6Al-4V sample, bulk properties regarding the shape, size and location of porosity defects can be detected on the micron scale.

\section{Experimental}

\subsection{Additive manufacturing- ORNL MDF 3D printing of the titanium cubes}

Samples of interest were electron beam-melted (EBM) in 2015 and 2016 from Ti-6Al-4V gas atomized powder. The build setup is shown in Fig. 1.

The powder is estimated to have a diameter ranging between 45 and $150 \mu \mathrm{m}$. Composition of the Ti-6Al-4V includes a majority titanium $(90 \%)$, aluminum $(6 \%)$, and vanadium (4\%), with trace amounts of iron, nitrogen, carbon and oxygen. An Arcam Q10 EBM machine printed layers from used powder in $0.05 \mathrm{~mm}$ height increments. A symbol, such as M3 or M5, is incorporated into one face of each cubic sample to distinguish samples. The beam spot size was not measured. For batch 4.2.75, the speed function of the Arcam Q10 system is $46 \mathrm{~mm} / \mathrm{s}$ and the focus Offset is $32 \mathrm{~mA}$. For batch, 4.2 .87 , the speed function is $30 \mathrm{~mm} / \mathrm{s}$ and the focus offset is $15 \mathrm{~mA}$. Hatch spacing of the system is $0.2 \mathrm{~mm}$. After the EBM printing is complete, the nonsintered powder is kept under a vacuum atmosphere, so that it can be used in future builds. Printing parameters for Ti6Al-4V samples are shown in Table 1 below. Similar AM printing procedures can be seen in [14-16].

\subsection{HZB CONRAD-2 imaging beamline}

Grating interferometry and high-resolution neutron imaging experiments were performed at the HZB BER II reactor neutron imaging instrument (CONRAD-2) [17, 18]. Highresolution imaging was performed without grating interferometry due to absorption of the beam by the gratings and to achieve higher resolution. A sample setup of the grating-based interferometry is shown in Fig. 2. 


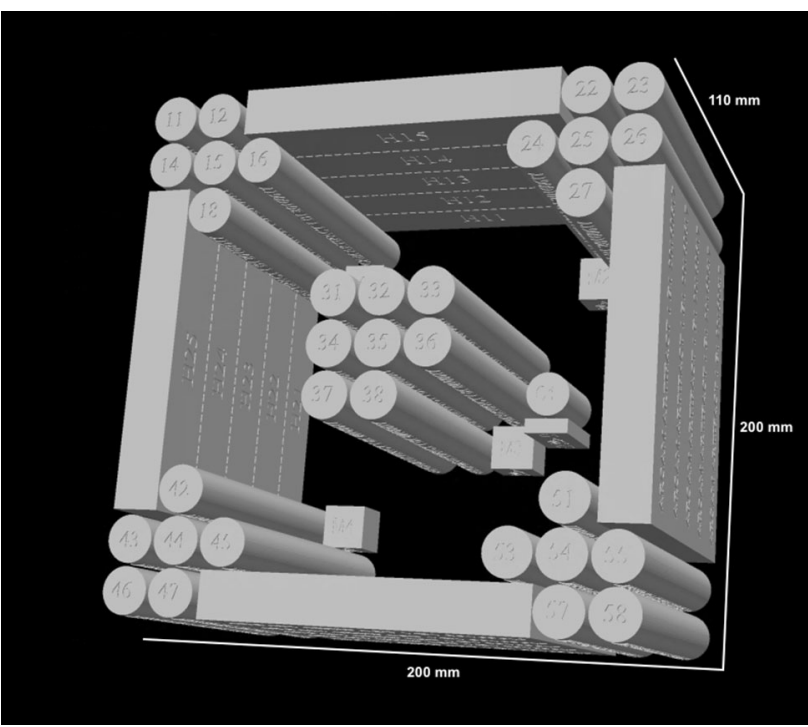

Fig. 1 Build fabrication of Ti-6Al-4V samples. Large rectangles measuring $105 \mathrm{~mm} \times 18 \mathrm{~mm} \times 23 \mathrm{~mm} \quad$ (Length $\times$ Width $\times$ Height), cylinders of $2 \mathrm{~mm}$ diameter and $105 \mathrm{~mm}$ height, and cubes of $(1.5 \mathrm{~cm})^{3}$ were printed using this setup of $200 \mathrm{~mm} \times 200 \mathrm{~mm} \times 110 \mathrm{~mm}$. Of interest in this neutron experiment are the cubic samples due to the small field of view at the neutron beamline $(6 \times 6 \mathrm{~cm})$

Table 12015 and 2016 Ti-6Al-4V Printing Parameters

\begin{tabular}{llll}
\hline Year & EBMC & Powder batch & Ti-6Al-4V pieces \\
\hline 2015 & 4.2 .75 & P942 & 52, H34-32, M1, M5 \\
2016 & 4.2 .87 & TEKNA Ti6Al4V & M3 \\
\hline
\end{tabular}

The beamline operated a pinhole of $3 \mathrm{~cm}$, allowing sufficient neutron flux $\left(2.4 \times 10^{7} \mathrm{n} \mathrm{cm}^{2} \mathrm{~s}^{-1}\right)$ at the detector ( $5 \mathrm{~m}, L / D$ value of 167 ). An Andor Ikon L-936 camera $(2048 \times 2048$ pixels, pixel size $30 \mu \mathrm{m})$ was used for imaging. The effective pixel size was $6.4 \mu \mathrm{m}$ for highresolution imaging and $30 \mu \mathrm{m}$ for interferometry. A 100 $\mu \mathrm{m}$ thick ${ }^{6} \mathrm{LiZnS}: \mathrm{Ag}$ scintillator was used to convert neutron flux into visible light.

In the grating interferometry setup, the source (G0), phase (G1) and analyzer (G2) gratings were placed at their respective first Talbot order distances. Grating position, alignment, and stability is critical for maintaining interferometer performance, as measured in percent visibility of a moiré pattern at the detector. In this experiment, the gratings were placed horizontal to the neutron beam to observe horizontal microstructure sensitivity in the samples. Alignment of the gratings began by observing the neutron beam with G0 (period of $790 \mu \mathrm{m}$ ) and G2 (period of $4 \mu \mathrm{m}$ ), where horizontal moiré fringes were observed. G2 was aligned close to the detector to eliminate highangle scattering from the sample. During the initial setup and alignment, G1 was the last grating to be installed; the

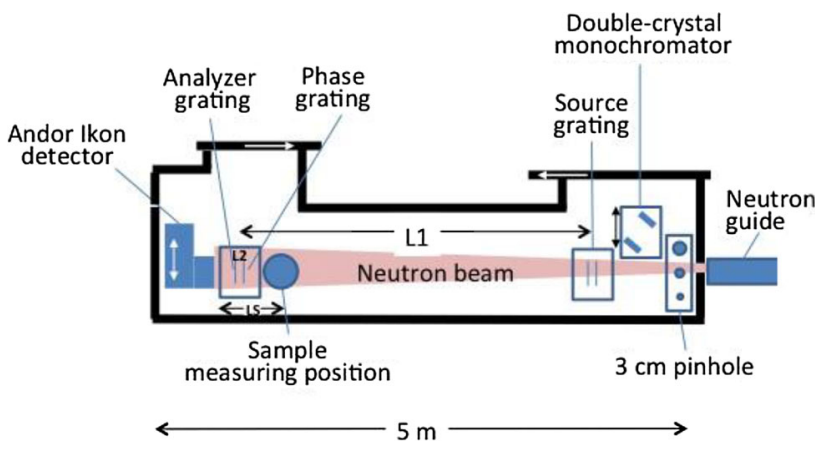

Fig. 2 Grating setup showing L1 of $4.78 \mathrm{~m}$ (G0-G1 distance), L2 of $2.27 \mathrm{~cm}$ (G1-G2 distance, first fractional Talbot distance for wavelength of $3.5 \AA$ ), and LS of $5 \mathrm{~cm}$ (sample-detector distance)

G0, G2 gratings and the detector were mutually aligned to give a low-frequency moiré pattern. The interferometer was operated in a stepped-grating mode, typically with twelve G1 positions evenly spaced over $12 \mu \mathrm{m}$, slightly more than the $7.96 \mu \mathrm{m} \mathrm{G1}$ period.

Interferometry and tomography imaging of two Ti-6Al$4 \mathrm{~V}$ cubes was performed with $20 \mathrm{~s}$ exposures, with one sample from 2015 (M5) and one from 2016 (M3). Highresolution attenuation tomography was performed on the 2016 cube, M3, after porosity was discovered with the gratings. An exposure time of $6 \mathrm{~s}$ provided good visibility (5\%) over 500 image projections. For tomography experiments, 181 steps from $0^{\circ}$ to $360^{\circ}$ were used.

\subsection{Neutron interferometry analysis}

A vectorized least squares algorithm was used for processing the raw images of the stepped-grating interferogram into projections of absorption, differential phase contrast, dark-field, percent visibility of the reference image, and $\chi_{v}^{2}$ of the sample images [19]. Across most of the sample interferograms, $\chi_{v}^{2}$ averaged near 2 , indicating an acceptable fit. The interferometer visibility in the reference images averaged $5 \%$ over the field of view, with the exception of a few damaged regions in the gratings. For regions in the reference visibility image with less than $3 \%$, a mask was generated and used to guide an inpainting correction to the projection [20]. Inpainting has characteristics similar to a median filter, but when guided by a visibility mask, it offers a more targeted image correction.

The tomography projections for attenuation and dark field were reconstructed with SIRT (ASTRA toolbox) [21]. The dark-field projections were reconstructed as ( 1 - dark-field) to set the air-region around the sample to zero. The reconstructed differential phase contrast volume was found to be essentially featureless. With some tomography runs producing 2534 projections, a dataset could result in well over 
17 GB. Binning of 2 or 4 was originally utilized for faster image processing. Cropping resulted in projections of 1.8 MB per image, or $4.7 \mathrm{~GB}$ per dataset.

\subsection{Visualization}

Three volumes were obtained and compared pairwise. Two volumes show neutron attenuation while the dark-field volume shows neutron small-angle scattering. The attenuation volume with pixel size of $6.4 \mu \mathrm{m}$ is regarded as the benchmark for feature detection; this volume is labeled A6.4. The attenuation volume collected simultaneously with the dark-field image has a pixel size of $60 \mu \mathrm{m}$ after binning of 2 and is labeled A60. The dark-field volume is labeled DF60.

Control points in the A6.4 and A60 volumes were manually selected in Avizo ${ }^{\mathrm{TM}}$ and used to generate a rigidbody affine transformation for the $\mathbf{A 6 0}$ and DF60 volumes. The high image contrast in A6.4 supported segmentation and morphological component analysis to provide a label field of the porosity structure (not shown). This label field was visualized as a skeletonization, Fig. 5.

\section{Theory}

To properly analyze the raw attenuation and dark-field datasets for the AM cubes, theory regarding the properties of materials and imaging must be understood. Attenuation images detect the sample composition based on neutron scattering and absorption. The dark-field image has a high connection with small-angle neutron scattering (SANS) and shows the microstructure of samples. The interferometer can be tuned to an auto-correlation length, $\zeta$, and the dark-field image can show the effect of features with dimensions at or smaller than $\zeta$. The theory behind quantitative neutron dark-field imaging has recently been published [22, 23]. A condensed explanation is presented here.

The real component of the neutron refractive index, $n$, is determined by

$n=1-\frac{\lambda^{2} N_{\mathrm{A}} b_{\mathrm{c}}}{2 \pi}=1-\frac{\lambda^{2}}{2 \pi}(\mathrm{NS} \mathrm{LD})$,

where $\lambda$ is the neutron wavelength, $N_{\mathrm{A}}$ is the number density, $b_{\mathrm{c}}$ is the coherent neutron scattering length, and NS LD is the neutron scattering length density as tabulated by NIST. ${ }^{1}$

The dark-field intensity (DFI) is dependent on the visibility of the beam, showing how intensity changes of the beam are generated by the phase grating, G1.

\footnotetext{
${ }^{1}$ NIST Neutron activation and scattering calculator; http://www. ncnr.nist.gov/resources/activation/.
}

$V=\frac{I_{\max }-I_{\min }}{I_{\max }+I_{\min }}$

When a sample is introduced into the beam path, modulations to the beam intensity can be detected at specific Talbot distances. The dark-field signal is a determination of sample visibility, $V^{\prime}$, over open beam visibility, $V$, as shown in Eq. 3.

$\mathrm{DFI}=\frac{V^{\prime}}{V}$

DFI is dependent upon several factors: period of the phase grating, $\mathrm{p}_{2}$, sample-to-detector distance, LS, and auto-correlation length, $\zeta$.

$\zeta=\frac{\lambda \times \mathrm{LS}_{\mathrm{eff}}}{p_{2}}$

To include sensitivity changes in the scenario of a sample being placed in front of G1 in the grating interferometer, the real sample-to-detector distance, LS $_{\text {eff }}$, must be accounted for. $L 1$ is the G0-to-G1 distance and $L 2$ is the G1-to-G2 distance. A large sample-to-detector distance implies the scattering angles probed are larger than those if the sample was placed close to the detector.

$\mathrm{LS}_{\mathrm{eff}}=\frac{(L 1+L 2-\mathrm{LS}) \times L 2}{L 1}$

For this experiment, $\lambda=3.5 \AA, \quad \mathrm{LS}=5 \mathrm{~cm}$, $\mathrm{LS}_{\text {eff }}=2.257 \mathrm{~cm}$, and $p_{2}=4 \mu \mathrm{m}$, giving a calculated autocorrelation scattering length of $\zeta=1.97 \mu \mathrm{m}$. The dark-field image gains intensity for features of this size or smaller.

\section{Results and discussion}

\subsection{Grating interferometry of Ti-6Al-4V cubes}

From the grating interferometry experiment, it was discovered that one of the AM cubes was not as uniform as expected. In both the attenuation and dark-field projections, a pore-like structure was observed in the 2016 cube (M3) but not in the 2015 cube (M5). Figure 3 shows the change in sample uniformity from 2015 to 2016 in attenuation and dark-field slices. In principle, the attenuation image can be used to measure the material density in the pores through the composition-weighted attenuation values, $\Sigma$, (see Table 2) and the dark-field can measure pore sizes through Eq. 4, the autocorrelation scattering length.

In determining the quality of the data, histograms revealed a slightly higher degree of correlation in the uniform cube (Fig. 4). While the porous cube has a similar range of attenuation values from 0.0028 to 0.0038 , the dark-field values are more obscure in the defective cube as 
seen by the lighter shade of orange above values of 0.001 . For the defective cube, the dark-field signal is much broader across the full attenuation range as compared to the concentrated intense signal in the good cube. This could indicate that even though the pores are microns in size (as compared to the $1.5 \mathrm{~cm}$ sample size), they greatly reduce the amount of sample scattering to behave more similarly to the environment.

\subsection{High-resolution imaging}

The main benefit of high-resolution attenuation imaging is obtaining roughly twice the resolution compared to grating interferometry. With an increase in effective pixel size from 30 to $6.4 \mu \mathrm{m}$, the pore structure of an AM sample can undergo further evaluation through segmentation and skeletonization. Skeletonization was found to be a valuable tool for observing porosity, where the connectivity of pores relates to the design parameters of the material. Figure 5 shows a skeletonization of the pore structure, ranging from radius sizes of 5-50 $\mu \mathrm{m}$.

Once a skeletonization showed the pore structure, it was necessary to visualize this structure in the sample. Figure 5 (right) shows the combination of skeletonization with the porous M3 cube. One great feature is the ability to observe the M3 symbol on the cube, indicating which face hosts the defects. In this case, the bottom face hosts the chimney

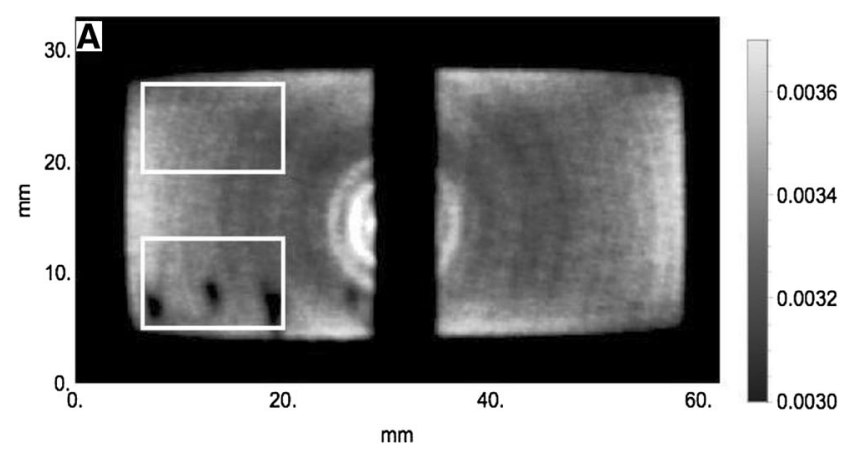

Fig. 3 a Attenuation and b dark-field slices from the grating interferometry A60 and DF60 volumes of Ti-6Al-4V cubes. The left cube is the 2016 M3 sample and the right cube is the 2015 M5, both $1.5 \mathrm{~cm}$ in length, width and height. Since attenuation and dark field are represented by visibility fractions in Eq. 3, there are no units for either term. Both the attenuation and dark-field images show the pore structure and shows how the pores build vertically across the sample. This information is highly valuable to the AM field to help alleviate any potential porosity issues during the printing or relaxation process.

Since the pore structure became more pronounced with high-resolution imaging, a new challenge arose to register Ti-6Al-4V cube volumes from differing experiments. With three known registration options (auto, manual, and landmarks), the best choice for the cubes was to utilize segmented data with correlation. The resulting Fig. 5 (left) shows a successful registration from the grating interferometry attenuation volume with the high-resolution attenuation volume. The two bounding boxes indicate the transformation applied to the interferometry dataset to register with the high-resolution dataset. The porous volume rendering of the high-resolution data is in white with the interferometry in yellow.

\subsection{Pore structure}

The attenuation and dark-field images provide complementary information about the pore structure. The attenuation image can have a high contrast to noise ratio, which allows binarization, segmentation, and skeletonization. The skeletonization defines the internal pore structure, yet makes the erroneous assumption that the pore/matrix interface is

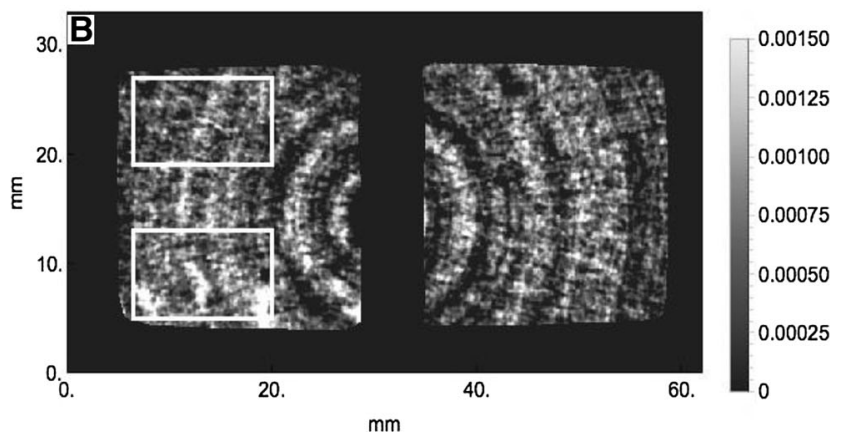

features of interest in the lower portion of the M3 sample. The white rectangles denote the regions analyzed with the histograms shown in Fig. 4. The color bars above are the same as the XY dimensions of Fig. 4. The ring artifacts are due to wear in the grating and detector system
Table 2 Ti-6Al-4V composition, thermal neutron scattering, absorption, and attenuation lengths $(\rho=4.43 \mathrm{~g} /$ $\mathrm{cm}^{3}$ )

\begin{tabular}{lclll}
\hline Element & wt\% & Scattering (barn) & Absorption (barn) & Weighted attenuation $\left(\mathrm{cm}^{-1}\right)$ \\
\hline $\mathrm{Ti}$ & 90 & 4.35 & 6.09 & 0.524 \\
$\mathrm{Al}$ & 6 & 1.503 & 0.231 & 0.010 \\
$\mathrm{~V}$ & 4 & 5.1 & 4.49 & 0.020 \\
$\Sigma\left(\mathrm{cm}^{-1}\right)$ & & & & 0.554 \\
\hline
\end{tabular}



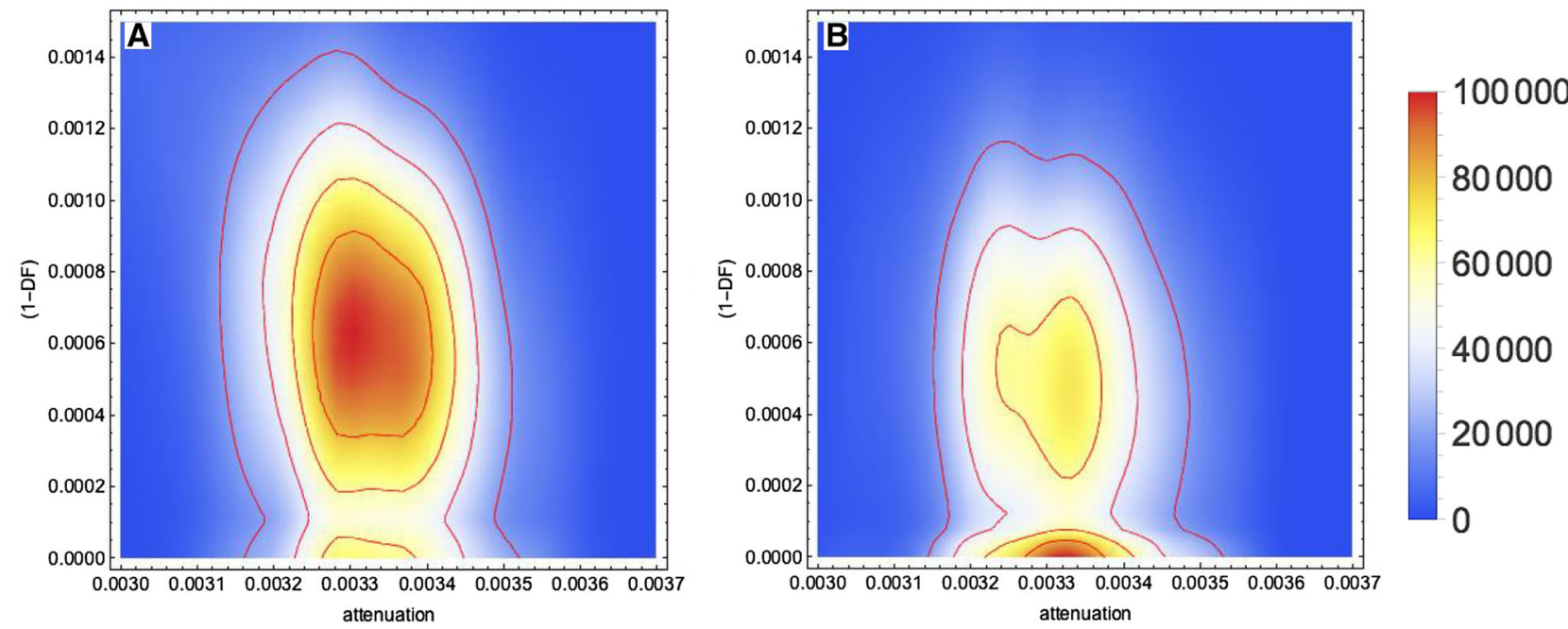

Fig. 4 Histograms of the M3 Ti-6Al-4V cube (A60 and DF60) for the portion with defects (a) and no defects (b). Defects are observed as reduced attenuation combined with increased neutron scattering

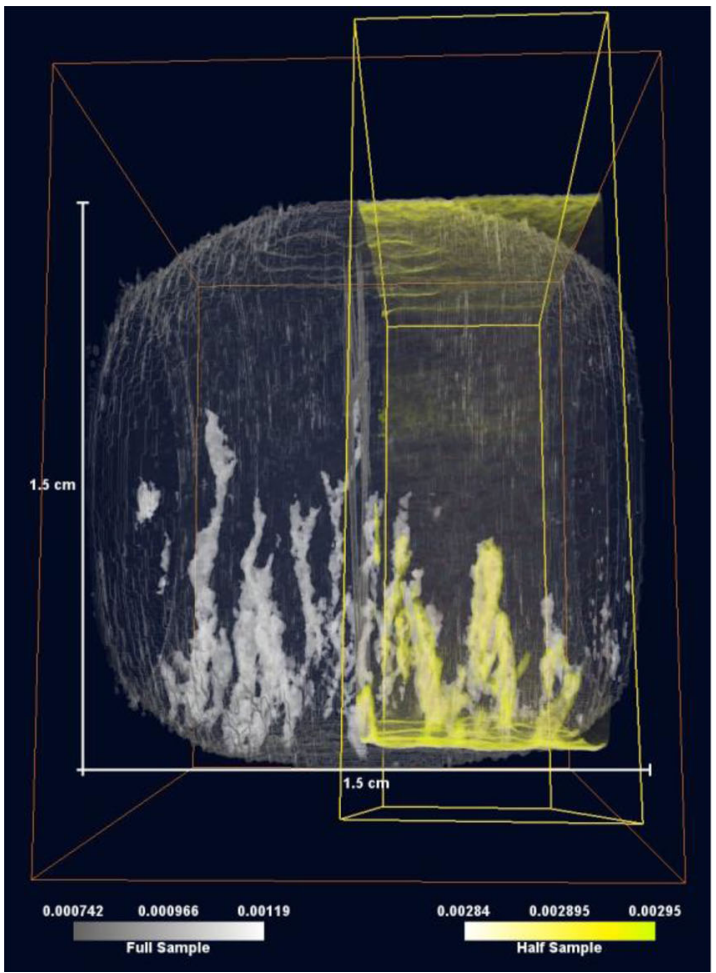

Fig. 5 On the left, the grating interferometry attenuation volume (yellow, A60) is registered to the high-resolution (white, A6.4) attenuation volume. Average values for the interferometry attenuation range from $0-0.00295$ to $0-0.00119$ for the high-resolution volume.

discrete. The dark-field image more accurately shows the range of porosity at the pixel-by-pixel level.

Shown in Fig. 6 is a pore observed with both interferometry and high-resolution attenuation imaging. With the high-resolution imaging, the pore has been segmented,

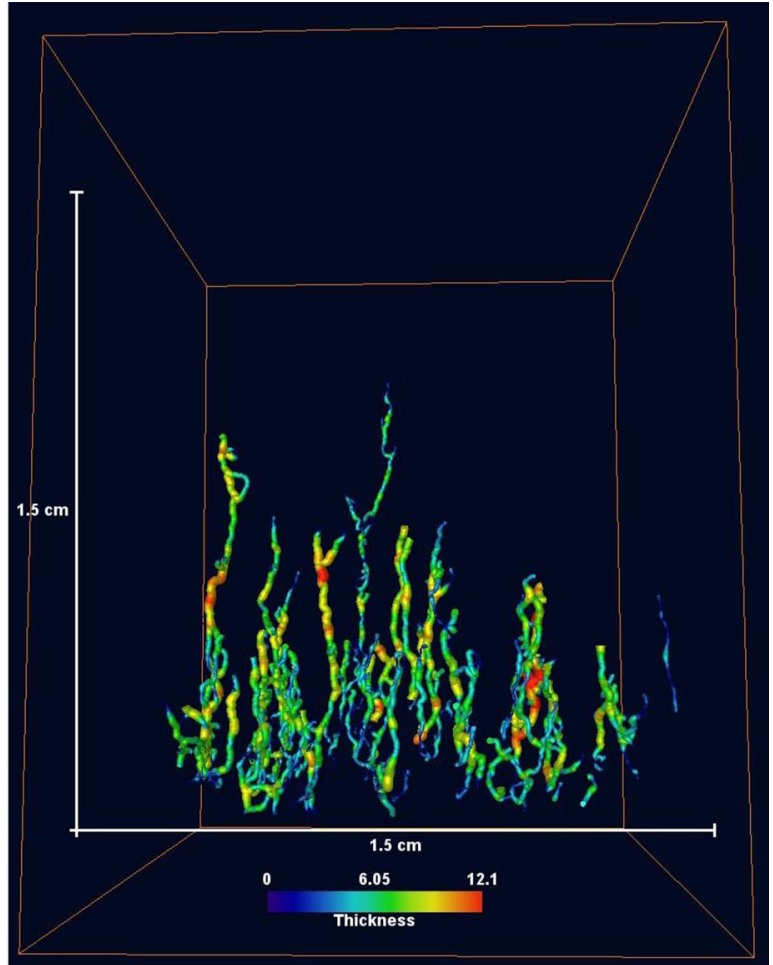

On the right, a skeletonization of the pore structure from A6.4. The pore segments are depicted in tubes scaled and colored by thickness (thickness min-max: 0.5-12.1, colormap: blue to red) (color figure online)

skeletonized (Fig. 5) and converted to a label field. The dark-field image, Fig. 6 d, shows the complicated structure in a pore. As the dark field is synonymous with small-angle scattering, brighter features are those regions exhibiting 

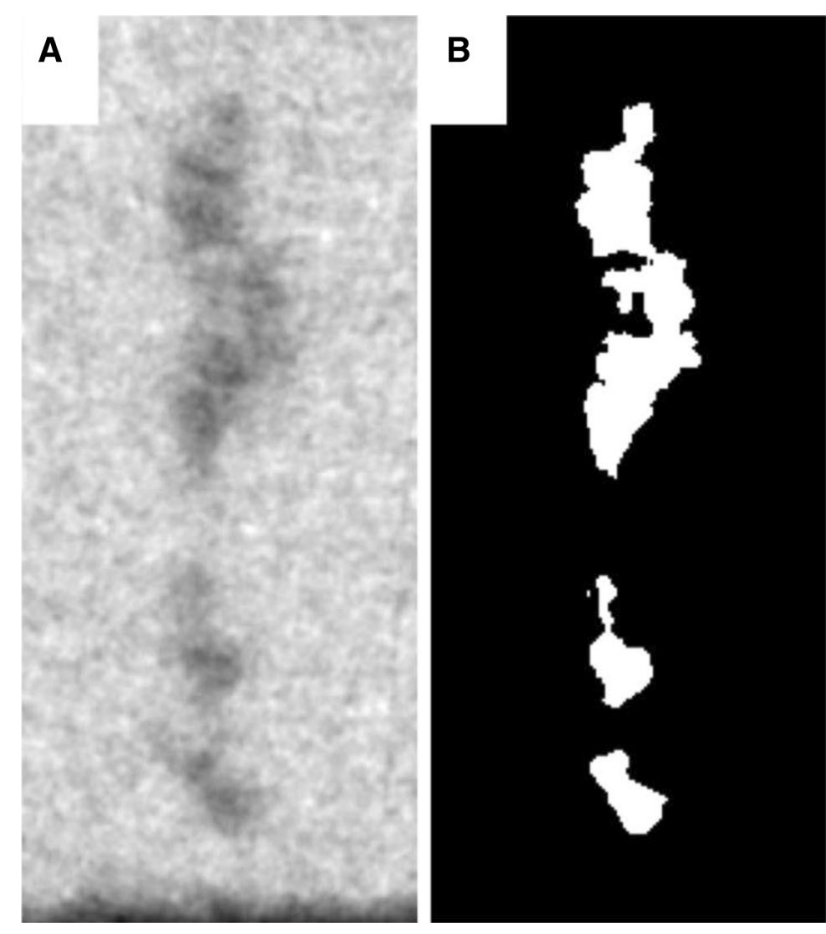

Fig. 6 A selected chimney-like structure shown with: a attenuation from a high-resolution tomography volume, A6.4, b binarized slice, c volume rendering of the binarization, and $\mathbf{d}$ the corresponding

strong scattering at the autocorrelation scattering length set by the interferometer.

Attenuation and dark-field reconstructions of the 2016 M3 sample exhibit a high amount of overlap in the chimney-like structure. Still, the dark field shows evidence of the feature outside the regions detected by attenuation. This suggests two different levels within the features. The larger chimney-like structures are highlighted by both attenuation and dark field.

The voids were originally treated as spherical; however, the discovery of the chimney structure helped to determine that the porosity is either a result of a lack of fusion or trapped gas. With the composition of $\mathrm{Al}$ rather small (only 10\%) in Ti-6Al-4V, it is difficult to determine if the pores are composed of aluminum or if the pores are truly voids in material. Pure aluminum could have attenuation values low enough to show up as a pore (as calculated in Table 2). However, the dark-field volumes disagree with this hypothesis as there is scattering from the pore structure at different locations than the attenuation volume. This likely indicates that the observed pores are a result of a lack of fusion (irregularly shaped pores) rather than keyhole or trapped gas (spherical voids). The attenuation values in the histograms in Fig. 4 are also above 0 , indicating that any voids are likely composed of material rather than gas.
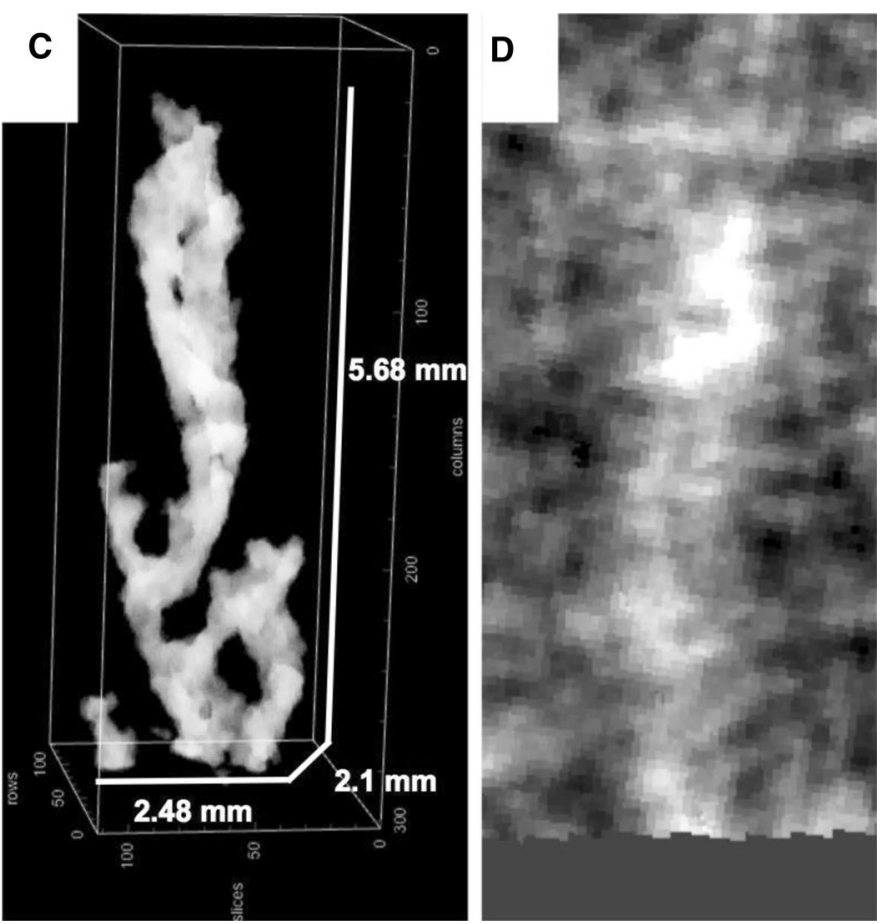

region from the interferometry experiment imaged with dark-field, DF60. The pore size is $2.5 \mathrm{~mm} \times 2.1 \mathrm{~mm} \times 5.68 \mathrm{~mm}$

\section{Conclusions}

A Ti-6Al-4V AM cube was imaged using neutron grating interferometry and high-resolution imaging. Synergistic analysis of high-resolution neutron tomography is achievable with lower resolution neutron interferometry/tomography. The dark-field image from neutron grating interferometry reveals sub-pixel scattering features at near micron dimensions. A comparison of neutron attenuation versus dark-field intensity shows that the chimney-like features attenuate neutron flux nearly as much as the bulk, but contain efficient neutron scattering sites with an autocorrelation length of $2 \mu \mathrm{m}$. There are two possibilities for the scattering differences: small gas-filled pockets present in the sample are less than $2 \mu \mathrm{m}$ or micron-scale phase separation of aluminum and titanium occurs in the material. If phase separation is causing the difference in scattering, the reduced neutron attenuation in the chimney-like features suggests this is due to aluminum enrichment within the chimney. A destructive test, like scanning electron microscopy, would show the true composition of the pores; however, further imaging experiments of the sample could no longer be explored.

This work shows one application of neutron grating interferometry for non-destructive evaluation of AM. Unfortunately, we note the HZB reactor is scheduled to close in 2020. The forthcoming VENUS beamline at the 
Spallation Neutron Source, Oak Ridge National Laboratory, USA could be an excellent site for grating interferometry.

Acknowledgements AJB and LGB gratefully acknowledge support from the Louisiana Consortium for Neutron Scattering (LaCNS), funded by the US Department of Energy- EPSCoR Cooperative Agreement No. EPS-1003897 and the Louisiana Board of Regents. MMK and RRD are supported by the Additive Manufacturing Demonstration Facility of Oak Ridge National Laboratory and by the US Department of Energy, Office of Energy Efficiency and Renewable Energy, Advanced Manufacturing Office, under contract DEAC05-00OR22725 with UT-Battelle, LLC. HZB is supported by the DOE Office of Science under contract DE-AC05-00OR22725 with UT-Battelle, LLC.

\section{Compliance with ethical standards}

Conflict of interest On behalf of all authors, the corresponding author states that there is no conflict of interest.

\section{References}

1. Sidambe AT, Todd I, Hatton PV (2016) Effects of build orientation induced surface modifications on the in vitro biocompatibility of electron beam melted ti6al4v. Powder Metall 59(1):57-65. doi:10.1080/00325899.2016.1153278

2. Ramakrishnaiah R, Al kheraif AA, Mohammad A, Divakar DD, Kotha SB, Celur SL, Hashem MI, Vallittu PK, Rehman IU (2016) Preliminary fabrication and characterization of electron beam melted Ti-6Al-4V customized dental implant. Saudi J Biol Sci. Ahead of Print. doi:10.1016/j.sjbs.2016.05.001

3. Vastola G, Zhang G, Pei QX, Zhang YW (2016) Modeling the microstructure evolution during additive manufacturing of ti6al4v: a comparison between electron beam melting and selective laser melting. JOM 68(5):1370-1375. doi:10.1007/ s11837-016-1890-5

4. Momose A (2005) Recent advances in X-ray phase imaging. Jpn J Appl Phys 44(9A):6355-6367. doi:10.1143/JJAP.44.6355

5. Wen H, Gomella AA, Patel A, Lynch SK, Morgan NY, Anderson SA, Bennett EE, Xiao X, Liu C, Wolfe DE (2013) Subnanoradian $\mathrm{X}$-ray phase-contrast imaging using a far-field interferometer of nanometric phase gratings. Nat Commun doi:10.1038/ ncomms 3659

6. Noda D, Tsujii H, Takahashi N, Hattori T (2008) Fabrication of $\mathrm{X}$-ray grating using X-ray lithography technique. ECS Trans 16(14):97-102. doi:10.1149/1.2992232

7. Grunzweig C, Pfeiffer F, Bunk O, Donath T, Kuhne G, Frei G, Dierolf M, David C (2008) Design, fabrication, and characterization of diffraction gratings for neutron phase contrast imaging. Rev Sci Instrum 79(5): art. no. 053703. doi:10.1063/1.2930866

8. Kim J, Lee SW, Cho G (2014) Visibility evaluation of a neutron grating interferometer operated with a polychromatic thermal neutron beam. Nucl Inst Method A 746:26-32. doi:10.1016/j. nima.2014.01.051

9. Thompson A, Maskery I, Leach RK (2016) X-ray computed tomography for additive manufacturing: a review. Meas Sci Technol 27(7):072001/1-072001/17. doi:10.1088/0957-0233/27/ $7 / 072001$
10. Cunningham R, Narra SP, Ozturk T, Beuth J, Rollett AD (2016) Evaluating the effect of processing parameters on porosity in electron beam melted Ti-6Al-4V via synchrotron X-ray microtomography. JOM 68(3):765-771. doi:10.1007/s11837-015$1802-0$

11. Cunningham R, Narra SP, Montgomery C, Beuth J, Rollett AD (2017) Synchrotron-based X-ray microtomography characterization of the effect of processing variables on porosity formation in laser power-bed additive manufacturing of Ti-6Al-4V. JOM 69(3):479-484. doi:10.1007/s11837-016-2234-1

12. Tremsin AS, Gao Y, Dial LC, Grazzi F, Shinohara T (2016) Investigation of microstructure in additive manufactured inconel 625 by spatially resolved neutron transmission spectroscopy. Sci Technol Adv Mater 17(1):324-336. doi:10.1080/14686996.2016. 1190261

13. Tremsin AS, McPhate JB, Vallerga JV, Siegmund OHW, Feller WB, Lehmann E, Kaestner A, Boillat P, Panzner T, Filges U (2012) Neutron radiography with sub-15 mu m resolution through event centroiding. Nucl Instrum Methods A 688:32-40. doi:10. 1016/j.nima.2012.06.005

14. Galarraga H, Lados DA, Dehoff RR, Kirka MM, Nandwana P (2016) Effects of the microstructure and porosity on properties of Ti-6Al-4V eli alloy fabricated by electron beam melting (ebm). Add Manuf doi:10.1016/j.addma.2016.02.003

15. Cakmak E, Kirka MM, Watkins TR, Cooper RC, An K, Choo H, Wu W, Dehoff RR, Babu SS (2016) Microstructural and micromechanical characterization of in 718 theta shaped specimens built with electron beam melting. Acta Mater 108:161-175. doi:10.1016/j.actamat.2016.02.005

16. Dehoff RR, Kirka MM, Sames WJ, Bilheux H, Tremsin AS, Lowe LE, Babu SS (2015) Site specific control of crystallographic grain orientation through electron beam additive manufacturing. Mater Sci Technol 31(8):931-938. doi:10.1179/ 1743284714Y.0000000734

17. Kardjilov N, Hilger A, Manke I, Woracek R, Banhart J (2016) Conrad-2: the new neutron imaging instrument at the HelmholtzZentrum Berlin. J Appl Crystallogr 49(1):195-202. doi:10.1107/ S1600576715023353

18. Kardjilov N, Hilger A, Manke I (2016) Conrad-2: cold neutron tomography and radiography at ber ii (v7). J Large Scale Res Facil 2(A1):1-6. doi:10.17815/jlsrf-2-108

19. Marathe S, Assoufid L, Xiao X, Ham K, Johnson WW, Butler LG (2014) Improved algorithm for processing grating-based phase contrast interferometry image sets. Rev Sci Instrum 85(013704):1-6. doi:10.1063/1.4861199

20. Bertalmio M, Vese L, Sapiro G, Osher S (2003) Simultaneous structure and texture image inpainting. IEEE Trans Image Process 12(8):882-889. doi:10.1109/TIP.2003.815261

21. van Aarle W, Palenstijn WJ, De Beenhouwer J, Altantzis T, Bals S, Batenburg KJ, Sijbers J (2015) The astra toolbox: a platform for advanced algorithm development in electron tomography. Ultramicroscopy 157:35-47. doi:10.1016/j.ultramic.2015.05.002

22. Betz B, Harti RP, Strobl M, Hovind J, Kaestner A, Lehmann E, Van Swygenhoven H, Gruenzweig C (2015) Quantification of the sensitivity range in neutron dark-field imaging. Rev Sci Instrum 86(12): art. no. 123704. doi:10.1063/1.4937616

23. Strobl M, Sales M, Plomp J, Bouwman WG, Tremsin AS, Kaestner A, Pappas C, Habicht K (2015) Quantitative neutron dark-field imaging through spin-echo interferometry. Sci Rep 5: art. no. 16576. doi:10.1038/srep16576 